\title{
Strategic Lean Organizational Design: Towards Lean World-Small World Configurations through Discrete Dynamic Organizational Motifs
}

\author{
Javier Villalba-Diez and Joaquín B. Ordieres-Meré \\ PMQ Research Group, ETSII, Universidad Politécnica de Madrid, José Gutierrez Abascal No. 2, 28006 Madrid, Spain \\ Correspondence should be addressed to Javier Villalba-Diez; javier.villalba.diez@alumnos.upm.es
}

Received 11 April 2016; Revised 20 June 2016; Accepted 13 July 2016

Academic Editor: Luis M. López-Ochoa

Copyright (C) 2016 J. Villalba-Diez and J. B. Ordieres-Meré. This is an open access article distributed under the Creative Commons Attribution License, which permits unrestricted use, distribution, and reproduction in any medium, provided the original work is properly cited.

\begin{abstract}
Organizations face strong international competition in the global market arena in achieving strategic goals such as high quality of product or service at lower cost while increasing their ability to respond quickly to requirements of the market. These challenges concern strategically designing organizations that can meet global challenges and specialize locally to meet performance constraints. After introducing the concept of organizational functional and structural motifs as small organizational building block, our findings suggest the hypothesis that a strategic organizational design (SOD) approach to meet these challenges involves maximizing the number and diversity of functional motifs, while minimizing the repertoire of structural motifs. By detecting characteristic structural motifs, we provide organizational leaders with specific Lean SOD solutions with which to meet local and global challenges simultaneously. As a matter of application, we show the implementation of such an SOD approach in nine US hospitals that form one large health care holding.
\end{abstract}

\section{Introduction}

Organizations face several strategic challenges in a global market arena such as demand for increasing product or service quality at lower costs with ever increasing agility [1]. Scholars have recently shown how market network structure affects its participants [2]. Under the multicontingency theory framework [3] where organizational structure follows strategy, these challenges act as forces upon organizations that counterreact by shaping their organizational configuration through strategic organizational design (SOD). There are authors [4] showing adopted structures as being positively related to the development of strategic flexibility and driving above-average returns in dynamic environments.

The state-of-the-art SOD theory distinguish two fundamental dimensions when discussing organizational configuration [5]:

(i) product/service/customer oriented with a strong outward orientation, (ii) functional specialization with a strong inward orientation, dividing the organization by specialized activities.

Depending on the focus, these scholars derivate qualitatively four basic organizational configurations: simple, functional, divisional, and matrix. These scholars however do not provide quantifiable metrics to dynamically steer the SOD process.

Organizational success can be made quantifiable through Lean Management metrics [6]. Lean Management can help the complex SOD process by providing a comprehensive framework that ought to help make SOD executable by means of the Lean Management paradigm.

Lean scholars $[7,8]$ suggest that in order to follow the Lean paradigm of systematic variability reduction [9], organizational structure should adapt a cross-functional value stream (VS) and customer oriented configuration [10]. However, with ever-changing functional requirements (see Figure 1), the cost of structural reconfiguration might 


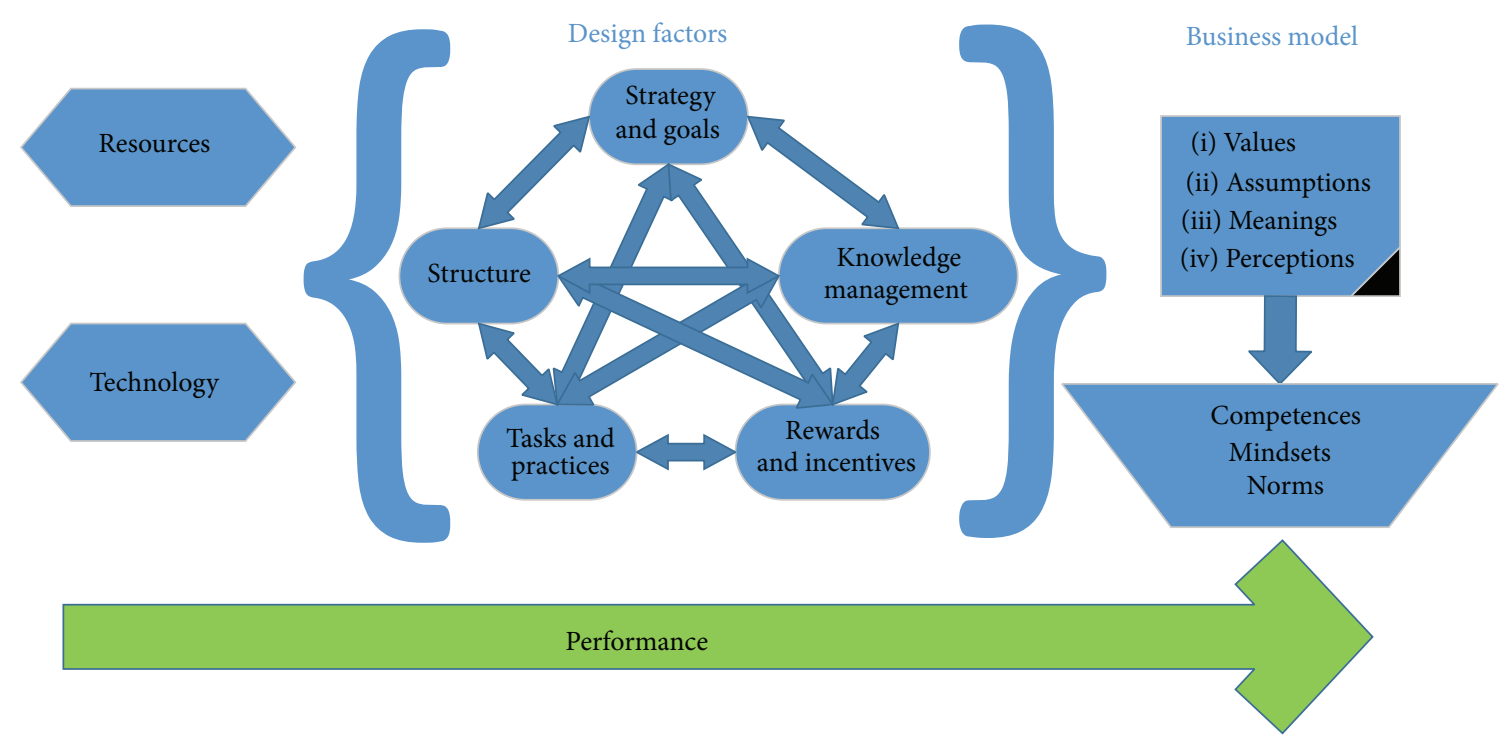

FIGURE 1: Organizational design model: relationship between organizational values and norms and design factors.

eventually be too high; it may increase the organizational resistance to change and the acceptance to Lean might ultimately sink [11].

The existence of other organizational dimensions within SOD is recognized [12]; however the authors focus on this paper upon the coexistence of structural and functional organizational dimensions, in order to provide a quantifiable structural and functional SOD frame that allows for a less costly and risky structure-function coexistence within the Lean transformation.

Organizations are complex systems [6] that, from an information exchange perspective [12], can be considered as networks under the "organizational network" paradigm [13]. A network is a set of objects called nodes or vertices that are connected together. The connections between nodes are called edges or links. In mathematics, networks are often termed graphs. Partitions of these graphs are referred to as subgraphs. One can formally define a graph as $G=(N, E)$, consisting of a set $N$ of nodes and a set $E$ of edges, which are ordered if the graph is directed. In this paper graphs are considered directed to indicate that information flows from one node to another. The diameter of a network $D$ is defined as the average distance between any two sites on the graph. The scaling of such diameter with the network size $N$ is highly relevant to phenomena such as diffusion, conduction, and transport, in this case of information, throughout the organizational network [14].

A network that presents long range global connections between highly connected nodes or hubs while presenting highly modular cluster configurations is called small world (SW) network [15]. Such networks present a high clustering coefficient (CC) and a small average path length (APL) which account for the topological properties already mentioned: a high CC means that the network tends to form clusters of highly dense connectivity, this serves for local efficient cliques, a small APL accounts for a small number of steps to connect distant agents, and this property helps gain global effectiveness and robustness to the overall network. The diameter $D$ of a SW network scales with the network size $N$ as $D \approx \ln (N)[14]$.

As its main contribution, this paper will propose Lean SOD. The latter is an organizational, structural, and functional model that helps in the development of a strategy to enable achievement of the Lean imperative and provides leaders with quantifiable metrics for its management. This will arise from the analysis of interactions at various levels of the organization that are considered to constitute a multiagent system that is represented as a network. Designing multiagent systems that can exhibit coherent group behavior based on a small number of simple rules is a very challenging problem $[16,17]$.

The adopted network representation of organizations will make analyzing specific patterns (structural building blocks or "motifs") possible [18]. Cutting edge state of the art in disciplines such as neuroscience [19, 20], biology [21], or industrial management [22] makes use of motifs to explain macroscopic characteristics of complex system's configuration.

The structure for the paper hereinafter continues with four phases: main contribution, management implications, case study, discussion, and conclusion. The contribution follows a clear roadmap: firstly structural and functional networks within organizations are defined. Secondly, few structural motifs that ought to provide organizational leaders with concrete Lean SOD solutions for achieving organizational challenges are characterized. Thirdly, several management implications of the author's findings are enunciated. Afterwards, the effects of the implementation of such an Lean SOD approach are shown in a case study performed within 9 US hospitals that form a health care corporation. Finally, the last section presents the conclusions from the research and its limitations and encourage further research in the field. By 
this approach, Lean SOD helps to merge Lean's executive level and SOD's great complexity.

\section{The Lean SOD}

A model that explains the design factors that impact organizational performance is presented in Figure 1. What is commonly understood about an organization is largely related to its performance. In turn, this is mainly the result of the interaction of different organizational designs, which depend on the specific inputs (resources and technology). When the organization faces significant changes, including changes in at design factors or potential changes in inputs, it will rebalance the relationships between those design factors and promote different common understandings. (This was previously known as organizational reconfiguration or restructuration.) The changes may impact the organization's strategy. This would affect its structure and practices.

In many cases, changes have a local impact (e.g., on a department, unit, or production facility). In some other situations, they have a global impact on the organization, as their pursuit of performance rise. Performance has three main dimensions, efficiency, effectiveness, and differentiation, and is directly related to the organization's business model. A business model is an expression of the logic behind the organization's creation and delivery of value. Implementation of a business model requires that top managers go beyond merely choosing the boundaries of the firm. They must articulate a vision, establish a culture, and create flexible organizational structures and incentives that support the desired organizational identity. A change in the design of an organization may lead to the recognition of new opportunities.

The existing literature enabled the links between organization design factors and competences to be identified. Therefore, the ability to successfully pursue new opportunities, while maintaining the existing business, was highlighted by [23]. Also, a specific organizational form that a multinational enterprise adopted influences the extent to which it can recombine and leverage knowledge gained across the enterprise [24]. In this particular perspective, networkoriented organizational representations enable relationships that transcend organizational boundaries, physical barriers, or hierarchical levels and can provide unique information and diverse perspectives to individuals who complete tasks in order to support a business model.

The literature also appears to suggest two different interpretations of the network organization. One interpretation is that of the organic organization. It is designed to handle tasks and to cope with environments that demand flexibility and adaptability. The other interpretation is that of a small central organization that relies on other organizations to conduct some of its business functions. The network organization can be viewed as a cluster of firms or specialized units that are governed by market mechanisms, instead of a strict chain of command. In order to handle organizational performance, a representation of the organization as the organic network is adopted in which formal communication/information relationships (links) among process owners (POs), who are denoted as nodes, are represented.

Although links between design factors and the business model have been identified, there is no information of how to enable dynamic reconfiguration of the structure to enact improvements in the business model leading to improvements in performance. This is particularly relevant, as dynamic capabilities are foundational to a business model. Organizational design also influences dynamic capabilities, such as the extent to which the organization can recognize factors that may require a change in the business model itself. Therefore, as can be expected, top managers become concerned with how to operationalize such relationships. The intrinsic value of this is evident, but no specific solutions have been provided previously. This paper proposes the Lean SOD mode for this purpose. It combines the following:

(a) The SOD framework that is provided in Figure 1.

(b) The Lean Management principles that enable organizations to "align value creation activities to the VS" [10].

(c) A network model of the organization that standardizes formal communication between POs.

(d) The Lean target for variance reduction in relevant KPIs [25].

In addition, the commitment of the POs will provide an automatic metric of the intrinsic capability of the existing configuration of design factors to improve the organization's performance. The remaining challenge is to determine the suitability of the organization to attain stability (Lean SOD configuration) in view of its dynamic evolvement.

Stability in performance requires a balance between local efficiency and global effectiveness. This is recognized as being essential for organizations [26]. SW structural organizational configuration allows for both advantages simultaneously. SW-ness is desirable in organizational networks in terms of SOD because this design characteristic allows for long range global connections between highly connected locally specialized clusters.

Under the information exchange paradigm in a complex system such as the brain, the CC measures "the efficiency of the local information transmission of every node" [27]. Similarly, in the brain a short APL "high global efficiency compared with the maximum efficiency of a random graph" [20]. It is therefore extrapolated that under the information exchange organizational paradigm a high CC increases local specialization hence fostering higher quality at lower cost and a short APL counts for an increased standardization speed and best practice sharing which potentially increases speed to market.

In line with [28], we emphasize the importance of patterning to produce topologies in organizations. The paper seeks to gain an understanding of SOD rules by investigating the organization's composition from smaller building blocks called "motifs." This will enable an assessment to be made of the configuration's suitability. The findings show that a large number of functional motifs are desirable in order to achieve organizational flexible and dynamic processing and that 


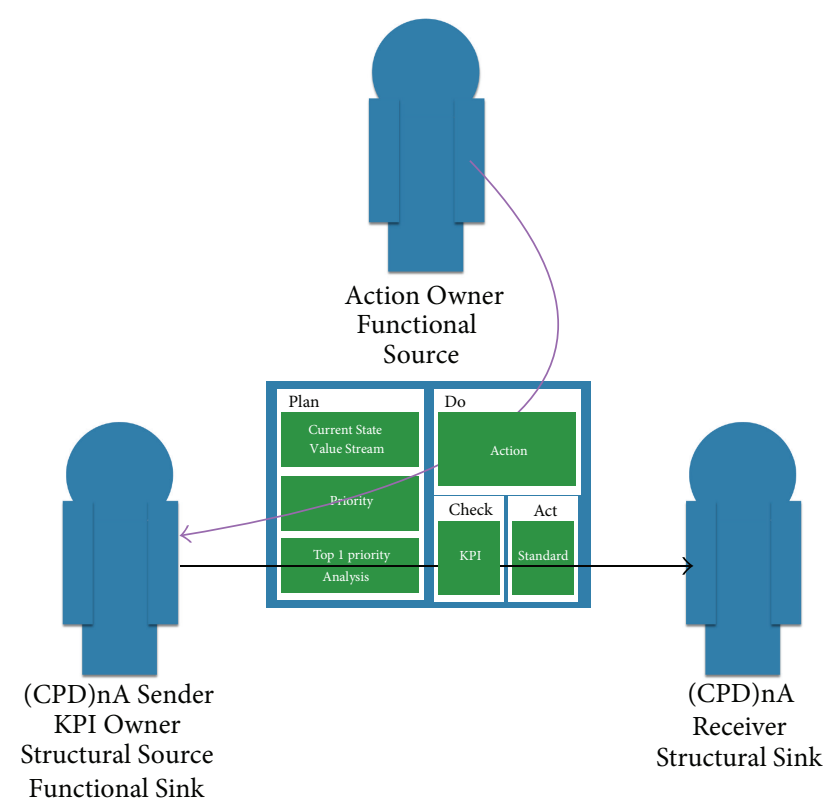

FIGURE 2: Representation of two nodes and one link from both LSN and LFN.

a small number of structural motifs ought to reduce SOD cost by promoting efficient encoding and assembly.

By proposing fundamental structural motifs, Lean SOD approach could avoid organizational leaders enormous change management costs due to reduced reconfiguration needs [11]. Furthermore, scholars [29] have shown that configuring networks for a small number of structural motifs and a high number of functional motifs simultaneously derives in SW structural configurations. Therefore, in addition to introducing the Lean SOD model, this paper characterizes the structural motifs that provide organizational leaders with clear structural building blocks to implement Lean SOD and attain SW-ness in the organizational configuration.

\subsection{Lean Structural and Functional Networks. The most} fundamental distinction between structural connectivity as physical "wiring diagram" and functional connectivity as web of "dynamic interactions" is borrowed from neuroscience [30] and adapted to the organizational SOD context. The physical information exchange wiring diagram that guides behavior is defined by how success is measured through KPIs at an organizational and individual level through VS performance indicators. The dynamic interaction between organizational agents is defined by their actions upon the VS. Therefore the two definitions of structural and functional networks, depicted in Figure 2, follow:

(i) We define Lean Structural Networks (LSN) as a set of nodes formed by process owners (POs) and edges formed by the KPI in the check phase of an interprocess communication standard (CPD)nA as described in [31] connecting the PO (CPD)nA Sender (Source) and the PO (CPD)nA Receiver (Sink). LSN are hence per definition directed networks. (ii) We define Lean Functional Networks (LFNs) as a set of nodes formed by POs and edges formed by the actions defined in the DO phase of the (CPD)nA connecting the $\mathrm{PO}$ responsible for the action (Source) and the PO (CPD)nA Sender (Sink).

These definitions have several SOD implications:

(1) Because there is a one-on-one relationship between $(\mathrm{CPD}) \mathrm{nA}$ and the structural edge (KPI), and this relationship does not exist between (CPD)nA and the functional edge (action), it implies that the LSN provides the substrate for LFN to exist. Therefore we focus on LSN when designing for SW-ness.

(2) It implies that the organizational goal-achievement system is embedded within the LSN. The reason for this is the nature of the LSN edges (the KPIs).

(3) It implies that the proper dimensioning of goals and reward systems lies within a balanced and "perceived as fair" LSN configuration and that this will impact organizational "tension" or organizational climate [12].

Once LSN and LFN have been defined, we aim to characterize organizational functional and structural motifs and identify core organizational structural motifs in order to provide organizational leaders with clear building blocks so as to perform Lean SOD.

2.2. Structural and Functional Network Motifs. Network theory applies to many types of networks. Networks that involve nontrivial topological features are designed as complex networks [20,32]. Most social and biological networks display such features, with patterns of connection between their elements that are not purely regular or purely random. Another aspect to consider is network dynamics, which usually evolve over time [33]. This includes complex technological networks, as depicted in [34]. They promote characterization of nonlinear behavior in two phase flows or the study of chaotic attractors. This facilitates the derivation of complex dynamic networks from time series, as they can embed different dynamics inside. This means that different topological properties can be derived. In those cases, the study of nontrivial patterns in the proposed network representations enables relationships to be established between the topological properties and pattern frequencies.

Network motifs were originally introduced to denote "patterns of interconnections that occur in complex networks at numbers that are significantly higher than those in randomized networks" [18]. Separating complex networks into a number of clusters helps to reveal specific, local properties [35]. Network motif, as another concept describing local properties of a network, is defined as a small connected subgraph that appears frequently and uniquely in a network. The size of a motif is given by the number of nodes it comprehends. Time evolution is reported in the literature to have returned to the same motifs again and again. This may be because they are the simplest and most robust circuits to perform these information-processing functions. This is the 
Motif size

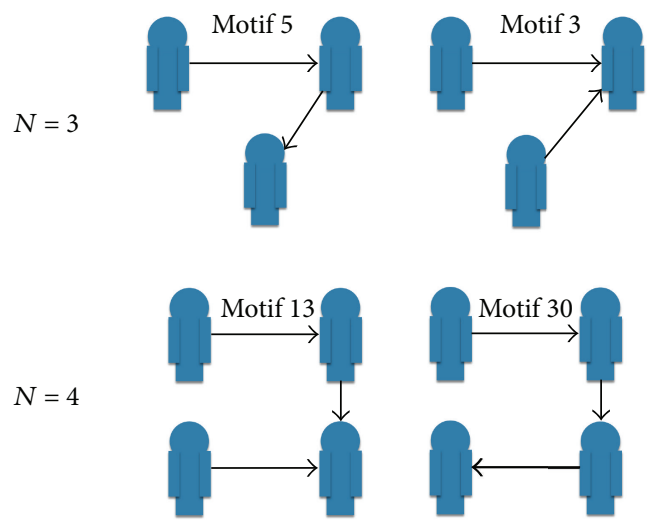

Most frequent structural motifs $N=3$ and $N=4$

FIGURE 3: Most frequent structural motifs depending on number of nodes involved $(N=3$ and $N=4)$.

exact the assumption that is behind the methodology that is proposed in this paper.

Following Sporns and Koetter's argumentation [29] our contribution will differentiate structural and functional network motifs:

(i) Organizational structural motifs (OSM) are the building blocks of LSN and consist of a subgraph of LSN of size NS.

(ii) Organizational functional motifs (OFM) are the building blocks of LFNs and consist of a subgraph of LFNs of size NF.

2.3. Implementing Lean SOD through OSM Characterization. One of the main advantages of Lean SOD is that it reduces structural reconfiguration costs towards strategic goals while keeping organizational functionality flexible. This is achieved by reducing the number of OSM while simultaneously increasing the number of OFM. Therefore, we propose certain OSM that support VS oriented SW configuration and suggest they have certain structural characteristics:

(1) The nodes in the OSM form a chain of feedforward interconnections along the VSs where the majority of nodes are highly integrated with their neighbours forming VS oriented clusters.

(2) In order to achieve SW-ness some nodes must have sparse long range connectivity. Therefore, we propose that the connections linking the end of the motifs are sparse and remain hence segregated while presenting long range connections with other clusters.

The combination of these two characteristics allow for a VS oriented SW and will derive in a Lean SOD configuration.

Reference [36] provides a motif taxonomy for $N=3$ and $N=4$. Under this taxonomy and considering previous characteristics, the most frequent structural Lean SOD motifs are represented in Figure 3.
As a result of this argumentation we propose three Lean SOD propositions and discuss their management implications.

\section{Lean SOD Discussion and Management Implications}

Proposition 1. In order to reduce reconfiguration costs by means of Lean SOD, the number of structural motifs ought to remain low. Therefore, the authors suggest that managers could simplify organizational structural connectivity by implementing the Lean SOD VS oriented motifs. The reduced number of the described Lean structural motif types will allow for reduced structural reconfiguration costs as well as for a VS oriented configuration.

The role of this proposition is to maintain structural configuration oriented towards the VS at a reduced cost by focusing solely on designing structural motifs with certain configurations.

Proposition 2. Functional flexibility increases maximizing the number of functional motifs. Therefore, one suggests that managers could complexify the organizational functional connectivity by allowing for a cross-functional diversification of functional motifs.

This proposition aims to increase the number of functional motifs within the structural substrate and this will allow for an increased computational and performance agility. This is in line with Ashby's "law of requisite variety" [37] as the variety of interactions in the stakeholder environment must increase with increasing environmental complexity.

The role of this proposition is to allow process owners to become more interconnected and therefore learn faster and with more flexibly. This is achieved because the organization 
is allowed to perform Lean actions across organizational divisions for instance.

Proposition 3. Lean SOD structural $S W$ configuration is achieved when $D S \approx \ln (N S)$. As shown by [29], structural $S W$-ness can be attained by implementing Propositions 1 and 2. Therefore, one suggests that managers steer the organizational structural configuration towards this mathematical equality by implementing Propositions 1 and 2.

The role of this proposition is to make Lean SOD quantifiable. This is necessary in order to implement and execute this Lean SOD strategy.

\section{Case Study}

In order to illustrate implementation of the Lean SOD method in an organization, a complete LFN and LSN of nine hospitals comprising a US health care corporation within one entrepreneurial group have been mapped in this case study. The case study also includes the corporate headquarter.

Following the recommendations of [38], we follow a clear case study roadmap. This roadmap has several phases: (1) scope establishment, (2) specification of population and sampling, (3) data collection, (4) data analysis, and (5) case closure.

4.1. Scope Establishment. We aim to study topological characteristics such as number and type of OSM and OFM as well as SW-ness of the selected organization. This study does not represent the dynamic evolution of the LSN and LFN but intends to represent the structural and functional characteristics of a given state and the consequences that can be derived from it.

The networks that model the health care holding under study are based on the paradigm of formal communication among various managers who follow the Lean concept and represent VS that are driven by interesting KPIs and their improvement as the fuel for the system (CPDnA, as described in [31]). The organization has 171 managers who are employed in nine hospitals in various states in the US and a corporate headquarters. These managers represent the nodes of the LSN and LFN being studied. The edges of the LSN are the KPIs that the managers report to each other and the edges of the LFN are the actions that are taken by the managers to serve certain processes. The LSN presents 346 edges and the LFN presents 975 edges at the moment of study. This means that the organization still has a huge potential for growth.

Data Collection. The health care holding implements (CPD)nA as described in [31] for two years in the moment of the data collection.

Due to the ratio of edges to nodes in both LSN and LFN, following the criteria given by [22], it can be ensured that the motif configuration of the LSN and LFN presented in this case can be considered mature for analysis, no matter the networks are still evolving. This means that the motif configuration has surpassed the initial growth evolutional phase and can be considered consistent.
In this context, a typical structural motif of $M=3$ represents three process owners connected by two (CPD)nA connections. This means that two of the process owners report (CPD)nA to another one of the process owners. For instance, in the example, two nurses report to a cardiologist on a certain (CPD)nA of a patient that has been recently operated on. This connection is cyclical and the two nurses are structurally linked with the doctor. A typical functional motif of $M=3$ represents three process owners connected by two actions performed in the DO phase of existing (CPD)nAs. This means that two of the process owners execute some action for the other two. For instance, in the example, the analytic laboratory department performs certain analyses for two doctors and report these actions to these two doctors. This connection is punctual and the technician responsible for the laboratory is linked functionally with the two doctors.

The LSN and LFN were mapped by analyzing an internal database where all (CPD)nA were stored. In order to avoid volatility in the data, this research only considered relevant connections in the LSN and LFN that endured for more than two months in the LSN and more than two weeks in the LFN.

Figure 4 provides a powerful visual of the overall systemic configuration of both LSN and LFN by representing the overall system of 9 hospitals linked together by a central corporate headquarter. Each link represents a process owner within the organization. Each structural link represents (CPD)nA and each functional link represents an action performed by one of the process owners within (CPD)nA.

Within-Case Data Analysis. As shown in Figure 5, the most frequent motifs of $M=3$ in the LSN are as expected Motif 5 and Motif 3. The presence of $M=3$ Motif 5 in the LSN indicates a high VS oriented Lean component. The most frequent motifs of $M=4$ in the LSN are Motif 13 and Motif 30. The presence of $M=4$ Motif 30 accounts for a high VS oriented Lean component. By reducing the number of structural motifs, structural reconfiguration cost remains low. Proposition 1 is therefore fulfilled.

As shown in Figure 6, while the diversity of OSM has remained low, the frequency of appearance of OFM is very much distributed (see Figure 6). By maximizing the number of functional motifs, the organization facilitated cross-functional diversification of knowledge and greater computing performance, in accordance with Proposition 2.

Following [29] if Propositions 1 and 2 are fulfilled, the authors expect an organizational structural network with SW characteristics. This is confirmed by the empirical data as $D \approx \ln (N)$ with an $R$-squared attachment of 0.977 for the structural networks of all hospitals and for the full network as shown in Figure 7. This confirms Proposition 3 because the organizations resulting from following Propositions 1 and 2 fulfill Lean SOD's Proposition 3 as well.

Case Study Closure. The case study has shown how, by reducing frequency of OSM and simultaneously increasing frequency of OFM, the configuration of LSN presents SW characteristics. This has potentially several benefits for organizational performance. 

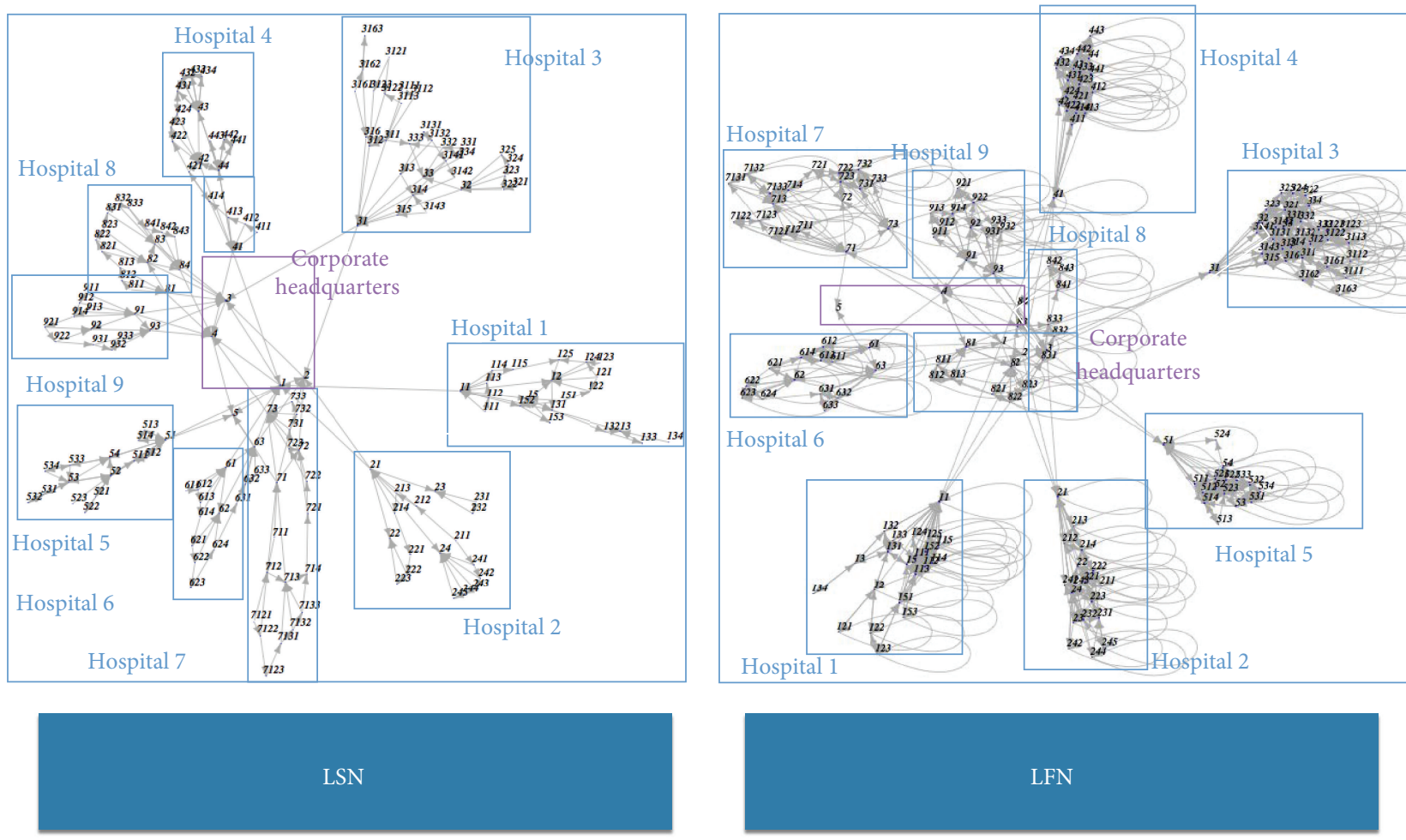

(a)

(b)

FIGURE 4: LSN on (a) and LFN on (b). They represent the full picture for the case study.

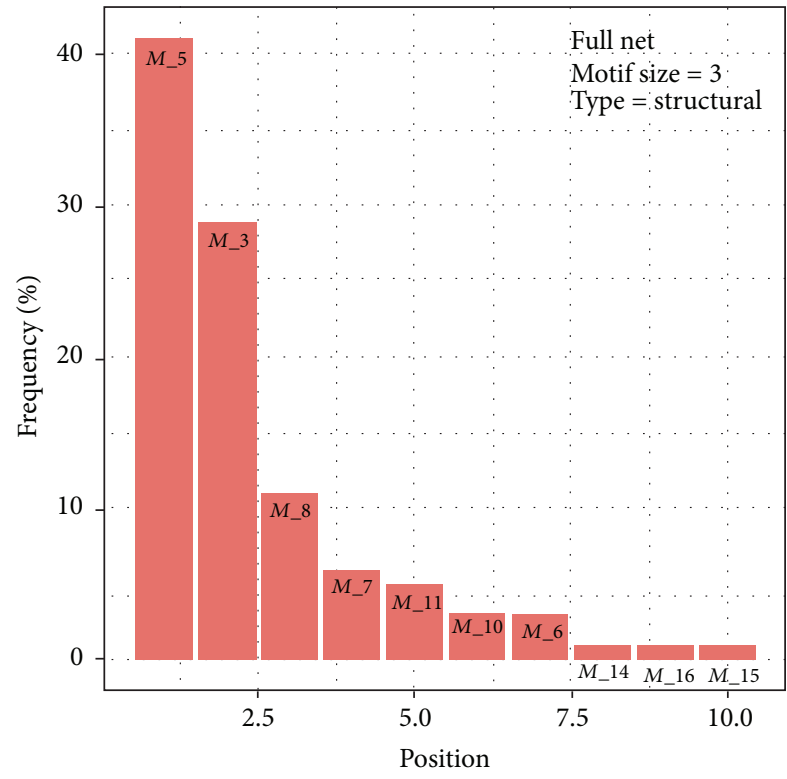

Most frequent OSM M $=3$ in LSN

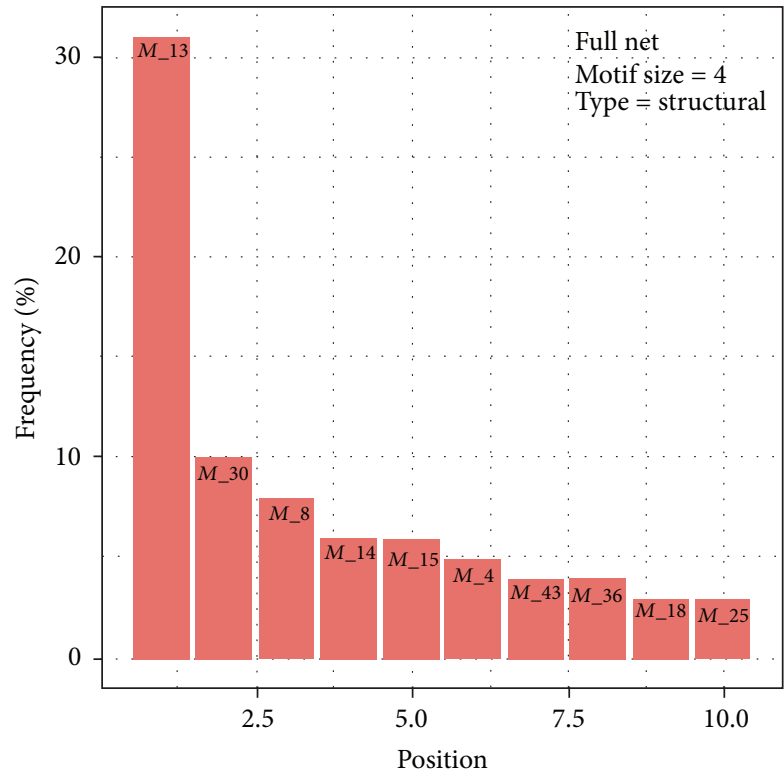

Most frequent OSM $M=4$ in LSN

FIgURE 5: OSM motif distribution: $M=3$ and $M=4$ in LSN. 


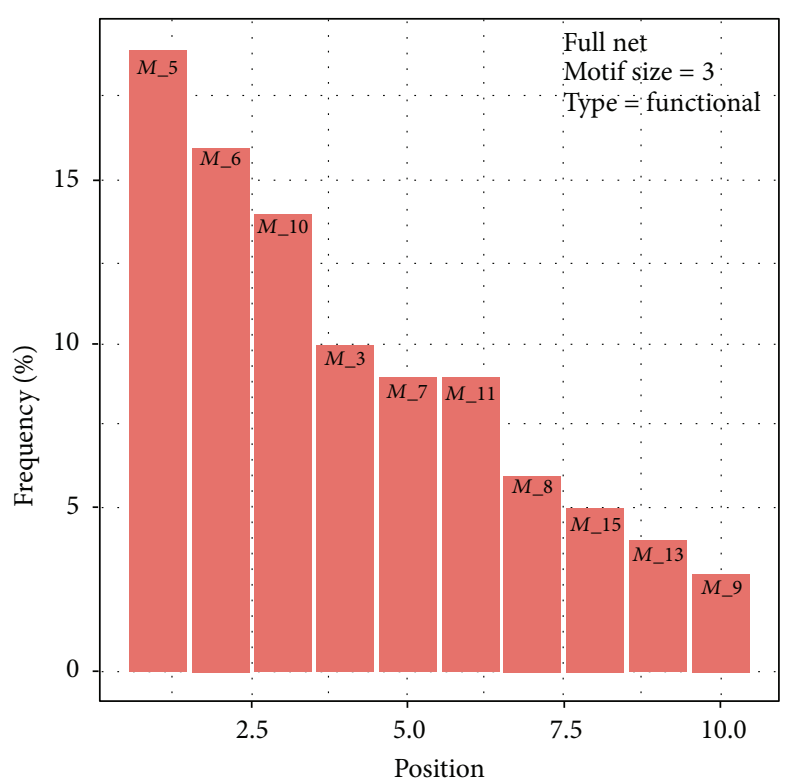

Most frequent OFM $M=3$ in LFN

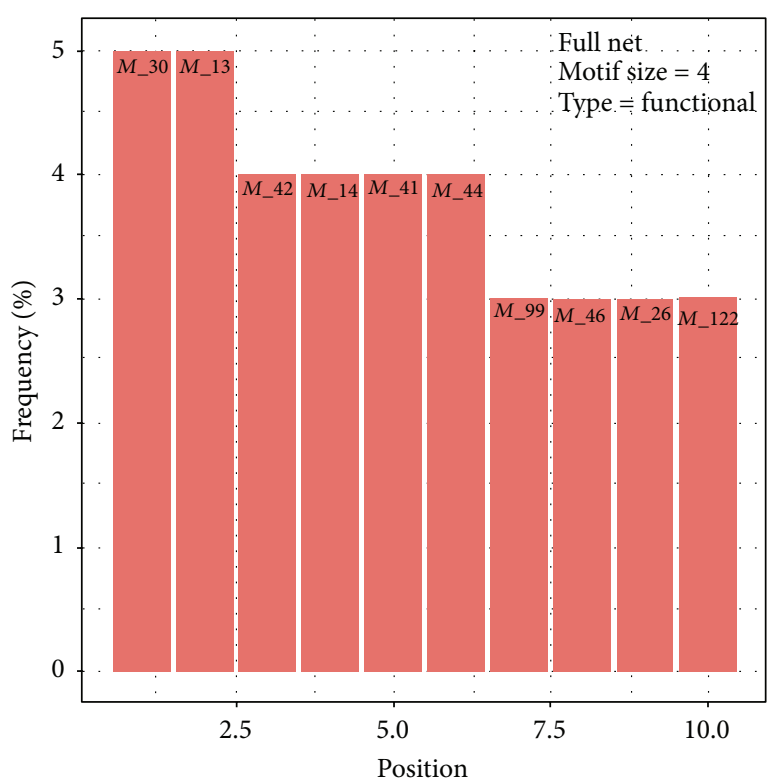

Most frequent OFM $M=4$ in LFN

FIGURE 6: OFM motif distribution: $M=3$ and $M=4$ in LFN.

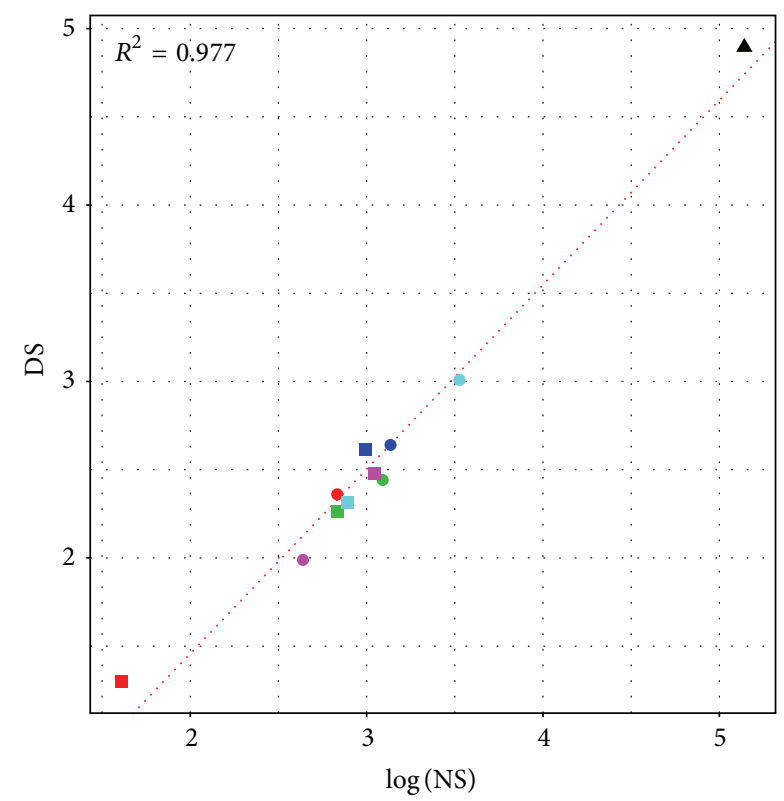

Type
A Full net
- Hosp. 1
- Hosp. 2
- Hosp. 3
- Hosp. 4
- Hosp. 5

FIGURE 7: SW architecture of LSN

By designing organizations with VS oriented structural motifs such as M 5 and M $3(N=3)$ and M 13 and $M 30$ $(N=4)$ Lean SOD allows for less costly structural costs.
By designing organizations with a wide range of functional motifs Lean SOD allows for high computational agility.

\section{Conclusions and Further Research}

This paper extends the published research by providing an operationalized way to link strategic organizational design to operational activities. It does in this way the Lean paradigm (the Lean SOD method). Indeed, it shows that motifs can provide a theoretical framework to bridge the communication gap between elementary components and macro properties of networks. (The organizational behavior of networks is revealed by the combination of individual motifs used in the transmission and transformation of information.)

Through the formulation of three management propositions, a novel way to link structural and functional motif configuration with potentially beneficial macro network properties such as SW-ness has been proposed.

Organizational motifs and their presented properties represent a configuration set that ought to provide organizational leaders with useful structural and functional Lean SOD characteristics that are expected to reduce organizational design related costs while increasing organizational performance. This will be aided by the strong relationships between organizational design factors and operational KPI values. Empowerment will be fostered throughout the entire system because of the intrinsic properties of CPDnA. All of those effects appear in a case study that involves several production units in different geographic locations.

Further research ought to expand this view by proposing Lean SOD integrating such motifs in effective dynamic ways that support organizational alignment of all organizational 
constituents. It is anticipated that the more complex network metrics as clustering coefficient entropy in this new research orientation will aid the analysis of the derived networks. The immediate effect of such a study will be the realization of the benefits of the Lean SOD method in performance and the assessment of the relationship between performance increases and network small worldness property.

\section{Competing Interests}

The authors declare that they have no competing interests.

\section{References}

[1] J.-G. Cegarra-Navarro, P. Soto-Acosta, and A. K. P. Wensley, "Structured knowledge processes and firm performance: the role of organizational agility," Journal of Business Research, vol. 69, no. 5, pp. 1544-1549, 2016.

[2] L.-R. Yang, J.-H. Chen, K.-S. Wu, D.-M. Huang, and C.-H. Cheng, "A framework for evaluating relationship among HRM practices, project success and organizational benefit," Quality and Quantity, vol. 49, no. 3, pp. 1039-1061, 2015.

[3] R. M. Burton, B. Obel, and G. DeSanctis, "Environment," in Organizational Design: A Step-by-Step Approach, p. 60, Cambridge University Press, Cambridge, UK, 2nd edition, 2011.

[4] M. Perez-Valls, J. Cespedes-Lorente, and J. Moreno-Garcia, "Green practices and organizational design as sources of strategic flexibility and performance," Business Strategy and the Environment, 2015.

[5] R. M. Burton, B. Obel, and G. DeSanctis, "Introduction," in Organizational Design: A Step-by-Step Approach, Cambridge University Press, Cambridge, UK, 2nd edition, 2011.

[6] M. Schneider and M. Somers, "Organizations as complex adaptive systems: implications of Complexity Theory for leadership research," The Leadership Quarterly, vol. 17, no. 4, pp. 351-365, 2006.

[7] J. P. Womack and D. T. Jones, "Introduction," in Lean (Thinking), p. 4, Simon \& Schuster, New York, NY, USA, 2nd edition, 2003.

[8] A. Haider and J. Mirza, "An implementation of lean scheduling in a job shop environment," Advances in Production Engineering \& Management, vol. 10, no. 1, pp. 5-17, 2015.

[9] R. Shah and P. T. Ward, "Defining and developing measures of lean production," Journal of Operations Management, vol. 25, no. 4, pp. 785-805, 2007.

[10] J. P. Womack and D. T. Jones, "Part II: from thinking to action: the lean leap," in Lean Thinking, p. 172, Simon \& Schuster, New York, NY, USA, 2nd edition, 2003.

[11] G. M. Levchuk, D. Serfaty, and K. R. Pattipati, "Section III: Organizational Level Adaptability. Normative design of projectbased adaptive organizations," in Understanding Adaptability: A Prerequisite for Effective Performance within Complex Environments, C. S. Burke, L. G. Pierce, and E. Salas, Eds., pp. 276-278, Elsevier, Amsterdam, The Netherlands, 1st edition, 2006.

[12] R. M. Burton, B. Obel, and G. DeSanctis, Organizational Design: A Step-by-Step Approach, Cambridge University Press, Cambridge, UK, 2011.

[13] R. L. Cross, J. Singer, S. Colella, R. J. Thomas, and Y. Silverstone, The Organizational Network Fieldbook: Best Practices, Techniques and Exercises to Drive Organizational Innovation and Performance, John Wiley \& Sons, 2010.
[14] R. Cohen and S. Havlin, "Scale-free networks are ultrasmall," Physical Review Letters, vol. 90, no. 5, Article ID 058701, 2003.

[15] D. J. Watts and S. H. Strogatz, "Collective dynamics of 'smallworld' networks," Nature, vol. 393, no. 6684, pp. 440-442, 1998.

[16] Y. Cao and Y. Sun, "Consensus of third-order multiagent systems with TIMe delay in undirected networks," Mathematical Problems in Engineering, vol. 2016, Article ID 6803927, 5 pages, 2016.

[17] F. Leon, "Emergent behaviors in social networks of adaptive agents," Mathematical Problems in Engineering, Article ID 857512, Art. ID 857512, 19 pages, 2012.

[18] R. Milo, S. Shen-Orr, S. Itzkovitz, N. Kashtan, D. Chklovskii, and U. Alon, "Network motifs: simple building blocks of complex networks," Science, vol. 298, no. 5594, pp. 824-827, 2002.

[19] L. L. Gollo and M. Breakspear, "The frustrated brain: from dynamics on motifs to communities and networks," Philosophical Transactions of the Royal Society of London, Series B: Biological sciences, vol. 369, no. 1653, 2014.

[20] D. Papo, J. M. Buldú, S. Boccaletti, and E. T. Bullmore, "Complex network theory and the brain," Philosophical Transactions of the Royal Society of London B: Biological Sciences, vol. 369, 2014.

[21] A. R. Kinjo and H. Nakamura, "Composite structural motifs of binding sites for delineating biological functions of proteins," PLoS ONE, vol. 7, no. 2, Article ID e31437, 2012.

[22] H. Shi and L. Shi, "Identifying emerging motif in growing networks," PLoS ONE, vol. 9, no. 6, Article ID e99634, 2014.

[23] Z. Simsek, "Organizational ambidexterity: towards a multilevel understanding," Journal of Management Studies, vol. 46, no. 4, pp. 597-624, 2009.

[24] C. A. O'Reilly and M. L. Tushman, "Ambidexterity as a dynamic capability: resolving the innovator's dilemma," Research in Organizational Behavior, vol. 28, pp. 185-206, 2008.

[25] D. M. Nguyen, "A new application model of lean management in small and medium sized enterprises," International Journal of Simulation Modelling, vol. 14, no. 2, pp. 289-298, 2015.

[26] J. Carneiro, S. B. Salter, and B. J. Punnett, "Local responses to global challenges: lessons from small economies," Journal of Business Research, vol. 68, no. 12, pp. 2588-2592, 2015.

[27] X. Di and B. B. Biswal, "Dynamic brain functional connectivity modulated by resting-state networks," Brain Structure and Function, vol. 220, no. 1, pp. 37-46, 2015.

[28] H. Mintzberg, The Structuring of Organizations, Prentice Hall, Engelwood Cliffs, NJ, USA, 1979.

[29] O. Sporns and R. Koetter, "Motifs of the brain," PLos Biology, vol. 2, pp. 1910-1918, 2004.

[30] O. Sporns, "Brain networks: structure and dynamics," in Networks of the Brain, pp. 31-50, The MIT Press, Boston, Mass, USA, 2011.

[31] J. Villalba-Díez and J. Ordieres-Meré, "Improving manufacturing performance by standardization of interprocess communication," IEEE Transactions on Engineering Management, vol. 62, no. 3, pp. 351-360, 2015.

[32] S. Boccaletti, V. Latora, Y. Moreno, M. Chavez, and D.-U. Hwang, "Complex networks: structure and dynamics," Physics Reports. A Review Section of Physics Letters, vol. 424, no. 4-5, pp. 175-308, 2006.

[33] Z.-K. Gao, Y.-X. Yang, P.-C. Fang, N.-D. Jin, C.-Y. Xia, and L.D. Hu, "Multi-frequency complex network from time series for uncovering oil-water flow structure," Scientific Reports, vol. 5, article 8222, 2015. 
[34] Z.-K. Gao, P.-C. Fang, M.-S. Ding, and N.-D. Jin, "Multivariate weighted complex network analysis for characterizing nonlinear dynamic behavior in two-phase flow," Experimental Thermal and Fluid Science, vol. 60, pp. 157-164, 2015.

[35] Z.-K. Gao and N.-D. Jin, "A directed weighted complex network for characterizing chaotic dynamics from time series," Nonlinear Analysis: Real World Applications, vol. 13, no. 2, pp. 947-952, 2012.

[36] J. A. Davis and S. Leinhardt, "The structure of positive interpersonal relations in small groups," in Sociological Theories in Progress, J. Berger, Ed., pp. 218-251, Houghton Mifflin, Boston, Mass, USA, 1972.

[37] J. R. Galbraith, “The future of organization design," Journal of Organization Design, vol. 1, no. 1, 2012.

[38] K. M. Eisenhardt, "Building theories from case study research," Academy of Management Review, vol. 14, no. 4, pp. 532-550, 1989. 


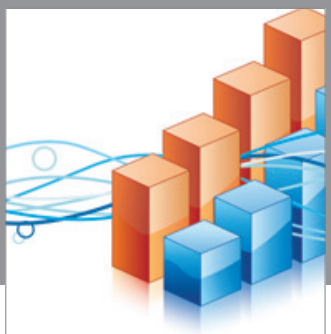

Advances in

Operations Research

vatem alat4

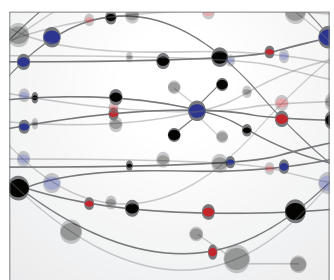

\section{The Scientific} World Journal
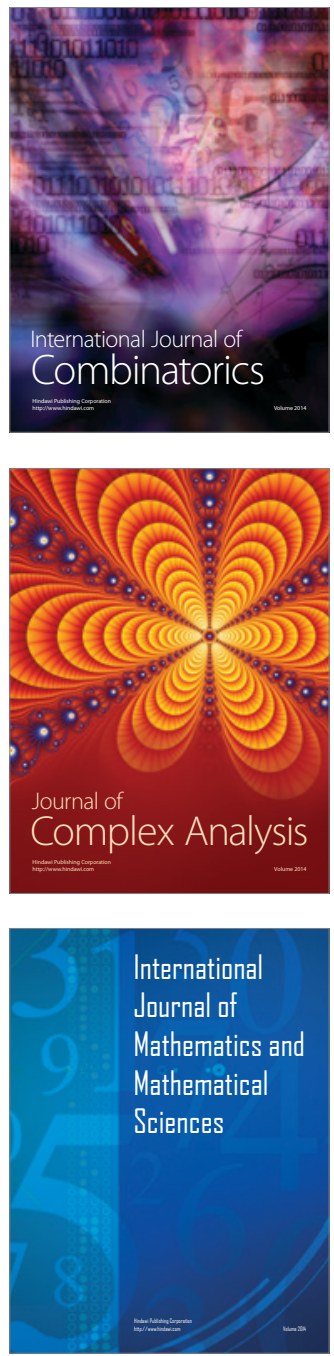
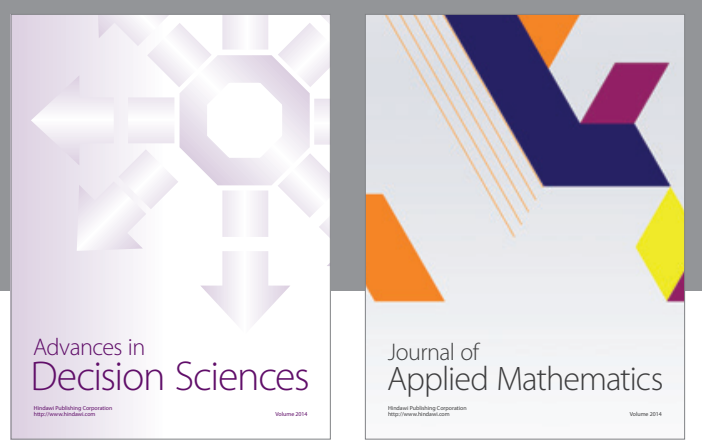

Algebra

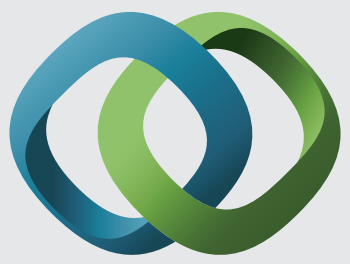

\section{Hindawi}

Submit your manuscripts at

http://www.hindawi.com
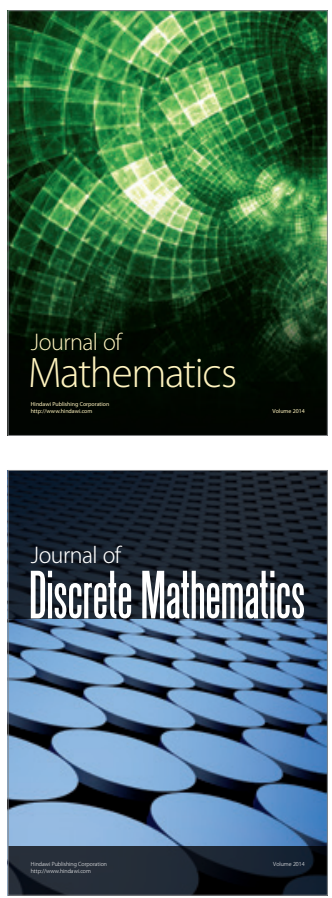

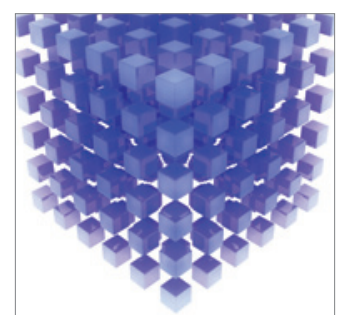

Mathematical Problems in Engineering
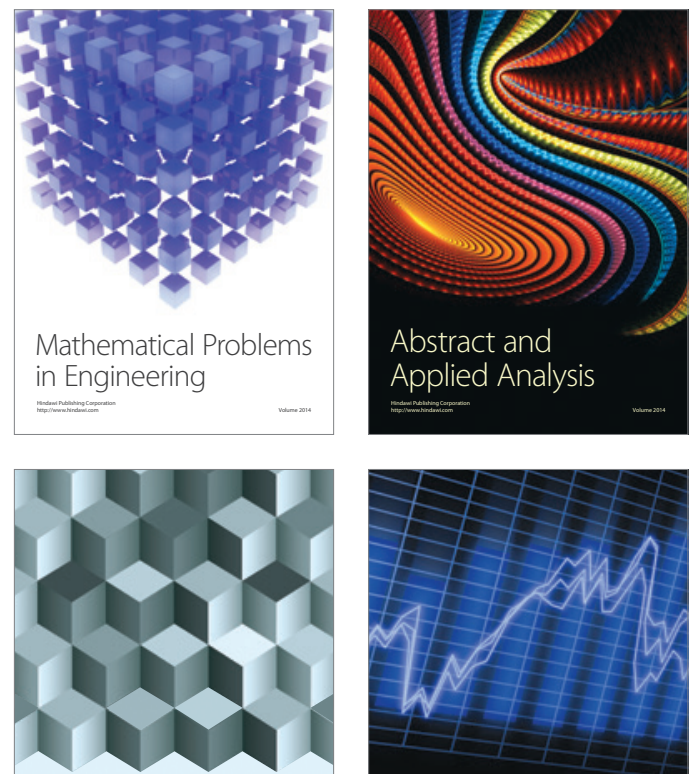

Journal of

Function Spaces

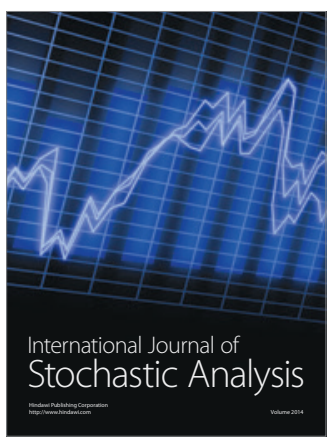

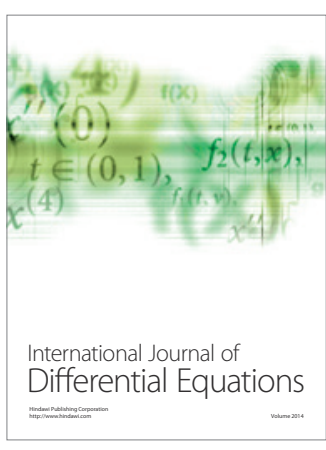
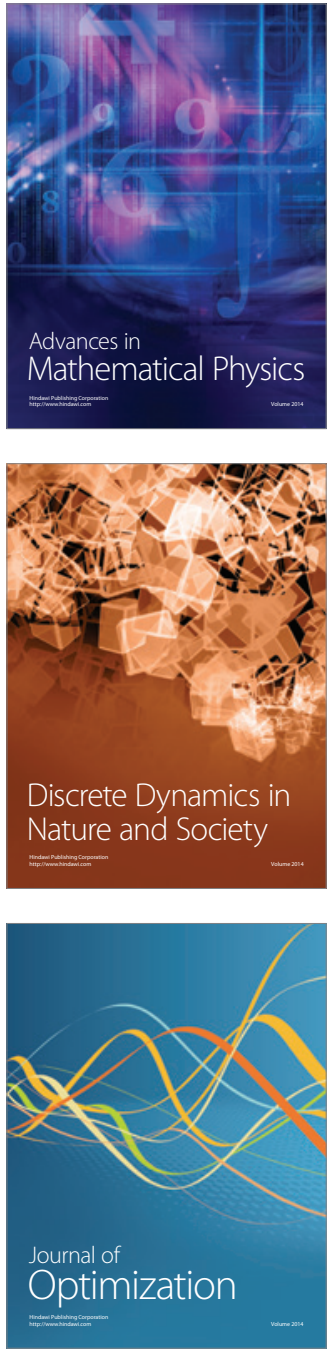